\section{Psychotherapie der Depression}

\author{
Einblicke in die tägliche Praxis
}

\section{A. Billeter}

Als Gegenstück zum Streit zwischen den Psychotherapierichtungen schildere ich Aspekte der üblicherweise praktizierten Psychotherapie. Dies auch im Hinblick auf das Suchen von "Guidelines» und auf die Diskussion um "Evidence-based Medicine».

In der Schweizerischen Ärztezeitung hat Kollege Zogg vier Konzepte der Depressionsbehandlung dargestellt, wie sie in diesen theoretischen Formen selten ausgeübt werden [1].

Wir Schweizer Psychiater/Psychotherapeuten arbeiten zum überwiegenden Teil eklektisch, $d$. h. wir benützen wohl hauptsächlich die Therapieform unserer intensiven Ausbildung, aber wir setzen je nach Patient und Situation Elemente verschiedener therapeutischer Schulen ein. - Die Entwicklung der Psychotherapie in den letzten 20 Jahren bestätigt uns darin, indem die neueren Therapieformen verschiedene Techniken aus einem breiten Spektrum von Ansätzen integrieren.

Von der ersten Therapiestunde an entwickeln sich parallel verschiedene Interaktionsstränge:

1. Anamnestisches Material und momentanes Befinden explorieren immer neu.

2. Begleiten, Anteil nehmen - nach Daniel Hell ("Welchen Sinn macht Depression?»): Aktives Eingreifen und geduldiges Zuwarten zeitgerecht miteinander verbinden.

3. Vermitteln von Information, Klären von Unsicherheiten, Erklären von seelischen Vorgängen.

4. Als psychoanalytisch ausgebildeter Therapeut versuche ich die Beziehung Patient-Therapeut dauernd $\mathrm{zu}$ fassen: Meist darf ich zu Beginn mit einer mild positiven Übertragung rechnen. Allerdings können schwer Depressive kaum affektive Beziehung aufnehmen; mein Beziehungsangebot kann verpuffen oder die Abwehr verhärten, später doch plötzlich lockern.

Im Laufe einer Therapie erwartet der Patient von mir vielleicht Entwertung und Ungeduld. Ich kann den Affekt ansprechen, evtl. die mir zugedachte Rolle annehmen, begrenzt mitagieren und mit einem Lächeln verfremden, sicher aber auch weiter schildern lassen und hinterfragen.

Korrespondenz:

Dr. med. A. Billeter

Hauptplatz 9

CH-8640 Rapperswil
5. Das Verhalten des Patienten wird gemeinsam reflektiert: z.B. wie kann er sich dazu bringen, täglich die Wohnung zu verlassen; z.B. welche Angehörigen könnten ihn unterstützen, was hindert den Patienten allenfalls daran. Solche Überlegungen führen häufig zu Hausaufgaben, z.T. schriftlich festgehalten, z.T. vom Patienten protokolliert.

6. Zur Depression gehören Einengung und Aggressionshemmung. Wie führe ich den Patienten zum Erleben seiner Gefühle? Ein flexibles Eingehen auf den einzelnen Menschen ist nötig. Mit dem Spüren der Gefühle fühlt er sich gestärkt und kann neu erleben und neu reagieren. Ein Patient kommt z.B. mit Suizidimpulsen und ohne Trauergefühle, welche in der ersten Besprechung dann unerwartet anklingen; in der zweiten berichtet er, drei Tage vorwiegend marschiert und viel geweint zu haben, jetzt aber wieder zu arbeiten. Die Medikamente bringt er zurück und 2 Monate später schreibt er mir von einem beruflichen Erfolg wozu in einem solchen Fall ein theoretisch fixiertes Therapieprogramm durchziehen wollen?

7. Angehörige ziehe ich bei schweren Depressionen nach Möglichkeit mit ein, bei leichten nur bei spezieller Indikation - und immer nur nach Besprechung mit dem Patienten. Angehörige können evtl. ihrerseits Unterstützung brauchen, sie helfen bei Informationen und bei Verhaltensänderungen, die Compliance kann bessern.

8. Antidepressiva und allenfalls zusätzlich symptomorientierte Medikamente sind bei schweren Depressionen unerlässlich. Bei leichteren wäge ich deren Einsatz mit dem Patienten ab.

Meistens lege ich in der 3. Stunde mit dem Patienten folgendes fest: Kann er sich durch meine Therapie überhaupt Hilfe versprechen? Welches ist unser Ziel? In welcher Weise werden wir arbeiten? Evtl. schlage ich einer Fokaltherapie entsprechend eine Stundenzahl vor, evtl. ist eine Begleittherapie sinnvoller. Jedem Gebildeten ist geläufig, dass im Leben Erfolgreiche auch bessere Chancen in einer Psychotherapie haben. Weniger bekannt und ebenso wichtig sind die (ebenfalls erforschten) Eigenschaften des Therapeuten: Für den Patienten einfühlbar positive Haltung seines Therapeuten, bei letzterem ein klares Konzept für den Fall und für die Arbeit (wobei ein Konzept gelegentlich revidiert werden muss), und eine klare Vermittlung von Ziel und Weg. Darum ist diese 3. Stunde so wichtig.

Psychoanalyse, systemische Denkweise, kognitive Ansätze usw. liefern mir die ganze Therapie hindurch laufend Modelle, welche erklären und welche zu neuen Möglichkeiten inspirieren. Immer wieder spielen auch Assoziationen hinein zur Psychopathologie, zur Entwicklungspsychologie (inkl. fortgeschrittenes Alter), zum selber Erfahrenen und Gelebten, mit zunehmender Praxis eindeutig mehr $\mathrm{zu}$ früheren Therapien. Das fachlich Gelernte ist somit nur ein 
Anteil, als Psychotherapeut bin ich unausweichlich auch meine Person.

Die verschiedenen Psychotherapieformen liefern mir auch unterschiedliche Techniken, welche sich zum Teil ergänzen. Dabei stosse ich erneut an Grenzen entsprechend meiner Ausbildung und wiederum meiner Person. - Immer neu bestätigt sich, Psychotherapie ist auch Begegnung zweier Menschen in ihrer Einmaligkeit, meine Person ist auch mein Werkzeug. Solche Individualität ist zeitweise beglückend, zeitweise ärgerlich, oft auch recht banal.

Ich möchte nicht missverstanden werden: Ich will nicht einer Beliebigkeit von Therapien das Wort reden. Gewisse Störungen verlangen entsprechende Schritte: Eine schwere Depression erfordert Psychopharmaka, in einer Suchtbehandlung sind Massnahmen zeitweise wichtiger als Psychotherapie, ein Paar mit Sexualstörungen wird ohne körperbezogene Übungen weniger profitieren, Zwangshandlungen bessern rascher mit einem Verhaltensprogramm. -
Wo nötig schicke ich den Patienten zu entsprechenden Instanzen: zur testpsychologischen Abklärung, zum Training bei mnestischen Störungen, zum Sozialarbeiter usw.

Aber ich glaube sagen zu dürfen, eine ausschliesslich auf einer Schulmethode basierende Psychotherapie bringt in der Regel weniger als die eklektische Arbeit; die gravierendsten Fehler sind mir bei rigidem Bemühen um eine Methode unterlaufen. - Wer Balint, Davanloo, Guntern, Hellinger usw. bei ihrer Arbeit erlebt hat, war wohl von den spezifischen Methoden fasziniert, doch mindestens ebenso von ihren umfassenden therapeutischen Möglichkeiten.

\section{Literatur}

1 Zogg W. Übersicht über einige Psychotherapien zur Depressionsbehandlung. Schweiz Ärztezeitung 2000; 81(37):2086-93. 